\title{
Nouvelle clause du Code de déontologie
}

\section{Caroline Hartmann}

Dre en droit, avocate, coresponsable du Bureau d'expertises de la FMH

Le Bureau d'expertises extrajudiciaires de la FMH s'est doté d'un nouveau règlement qui entrera en vigueur le $1^{\text {er }}$ octobre 2019 [1] et réglera la répartition des coûts entre les parties. De son côté, le Code de déontologie s'est vu ajouter une nouvelle clause concernant la rémunération des experts. Le présent article a pour but de faire connaître cette nouvelle clause et d'expliquer ses conséquences pour les membres de la FMH.

Pour pouvoir faire la lumière sur les faits médicaux et, partant, sur une éventuelle violation du devoir de diligence du médecin, une expertise médicale est en principe nécessaire. La FMH s'est dotée en 1982 d'un Bureau d'expertises extrajudiciaires chargé d'examiner ces cas. En vertu de la décision de la Chambre médicale du 24 juin 1993, les membres de la FMH sont tenus de se soumettre aux procédures d'expertise extrajudiciaire de la FMH [2].

\section{Prestations de l'assurance responsabilité civile en cas de sinistre}

Lorsqu'un patient fait valoir des prétentions en dommages-intérêts envers son médecin traitant, l'assurance responsabilité civile (RC) professionnelle a pour fonction d'une part d'indemniser le patient, dans la mesure où ses prétentions sont fondées, et d'autre part de protéger le médecin contre les prétentions infondées (protection juridique passive).

\section{L'assurance RC professionnelle indemnise} les patients dont les prétentions sont fondées et protège les médecins contre les prétentions infondées.

1 Les membres de la FMH sont tenus de se soumettre aux procédures d'expertise extrajudiciaire de la FMH. Si elle est membre de l'ASA, leur assurance est tenue de prendre en charge les honoraires des experts. pertise font partie des coûts du sinistre - ou cela peut relever de la protection juridique passive, pour se prémunir contre des prétentions infondées.

\section{Gentlemen's agreement}

Un "gentlemen's agreement» a été passé il y a 37 ans entre la FMH et l'Association suisse d'assurances (ASA). Les assureurs membres de l'ASA couvrent sans réserves les honoraires des experts sur la base de ce "gentlemen's agreement», soit indépendamment du résultat de l'expertise et quelle que soit l'opinion de l'assureur quant à l'utilité d'une expertise de la $\mathrm{FMH}^{1}$.

Les assureurs membres de l'ASA couvrent les honoraires des experts sans émettre de réserves.

\section{Une couverture des coûts lacunaire}

La couverture des coûts n'est cependant pas garantie dès lors que l'assureur n'est pas membre de l'ASA et qu'il ne fournit pas de garantie de paiement. Par le passé, du fait de cette couverture lacunaire des coûts, le Bureau d'expertises extrajudiciaires n'était parfois guère en mesure de mettre en œuvre une expertise. Dans ces cas, le patient avait alors la possibilité de demander une expertise privée à ses frais ou d'engager une procédure judiciaire. Quand cela est possible, il est toutefois préférable d'éviter les procédures judiciaires, vu la difficulté à apporter des preuves et le risque pour les parties de devoir en supporter les frais, sans oublier la longue durée des procédures, qui représente une charge pour toutes les parties impliquées. 
2 Les disciplines médicales le plus souvent concernées extrajudiciaire de la FMH sont la chirurgie, la chirurgie orthopédique et la traumatologie de l'appareil locomoteur, la médecine interne générale (y c. médecine de famille), ainsi que la gynécologie et lobstétrique. La FMCH a conclu un contrat-cadre avec la Bâloise Assurances (membre de l'ASA) pour que les membres de la FMH avec activité chirurgicale et invasive impliquant des risques plus élevés de violation du devoir de diligence puissent conclure un contrat d'assurance RC répondant à leurs besoins. 3 La société de discipline médicale concernée livre une première appréciation médicale du cas. par une expertise

\section{Art. 35 al. 2 Code de déontologie de la FMH}

Suite à la décision de la Chambre médicale du 9 mai 2019, les membres de la FMH seront nouvellement tenus, en vertu du droit associatif, de prendre en charge les honoraires des experts lorsque leur assurance RC professionnelle n'est pas membre de l'ASA et qu'elle n'accorde pas de garantie de paiement.

\section{Art. 35 al. 2 (nouveau) Code de déontologie}

"Le médecin est tenu de se soumettre à une expertise du Bureau d'expertises extrajudiciaires de la FMH. Dans la mesure où l'assureur responsabilité civile ne fournit pas de garantie de paiement, le médecin est tenu de prendre à sa charge les honoraires de l'expert mandaté par le $\mathrm{Bu}$ reau d'expertises extrajudiciaires de la FMH.»

\section{Un risque financier faible pour les membres de la FMH}

Le risque financier supporté par les membres de la FMH reste faible, malgré cette nouvelle disposition déontologique. La majorité des assureurs RC sont affiliés à l'ASA et donc liés par le "gentlemen's agreement $»^{2}$. L'ASA compte environ 80 compagnies d'assurance de petite ou de grande taille, actives en Suisse ou à l'échelle internationale [3].

\section{Le risque financier supporté par les membres de la FMH est faible.}

De plus, toutes les réclamations de patients ne conduisent pas à une expertise de la FMH. Si un cas s'avère d'emblée sans espoir, l'avocat du patient ou l'organisation de patients qui le défend ne vont vraisemblablement pas engager de procédure. Par ailleurs, le Bureau d'expertises de la FMH n'entre en matière que lorsqu'il existe suffisamment d'indices d'une violation du devoir de diligence médicale ${ }^{3}$.

\section{Toutes les réclamations de patients ne conduisent pas à une expertise de la FMH.}

Mentionnons toutefois que si les membres de la FMH sont tenus de se soumettre à une expertise extrajudiciaire de la FMH, les parties impliquées sont libres de mandater le bureau d'expertises de leur choix.

\section{Délai transitoire pour l'entrée en vigueur de l'art. 35 al. 2 du Code de déontologie}

La nouvelle réglementation de l'art. 35 al. 2 du Code de déontologie de la FMH entrera en vigueur le $1^{\text {er }}$ avril
2020. Cette entrée en vigueur différée a pour but de ménager un délai transitoire durant lequel les médecins pourront procéder aux vérifications qui s'imposent auprès de leur assureur responsabilité civile professionnelle et, au besoin, adapter leur couverture d'assurance.

L'art. 35 al. 2 du Code de déontologie de la FMH entrera en vigueur le $1^{\text {er }}$ avril 2020.

\section{L'assurance RC professionnelle}

La FMH vous recommande de contrôler régulièrement votre couverture d'assurance responsabilité civile, en vous posant notamment les questions suivantes:

- La somme d'assurance est-elle d'au moins 10 millions de francs?

- Tous vos domaines d'activité sont-ils bien couverts?

- Avez-vous conclu une assurance subséquente en cas de cessation d'activité pour toute la durée des délais de prescription légaux?

- Votre assureur RC est-il membre de I'ASA?

Vous recevrez un conseil compétent auprès de FMH Insurance Services [4].

\section{Conclusions}

Tous les assureurs RC professionnels actifs en Suisse ne sont pas membres de l'ASA, raison pour laquelle certains ne sont pas liés par le "gentlemen's agreement». Ces assureurs acceptent certes au cas par cas d'examiner une prise en charge des coûts, mais l'expérience montre qu'ils le font rarement sans émettre de réserves.

Dans les cas où la police d'assurance RC ne prévoit pas de couverture des honoraires d'experts et où la compagnie d'assurance n'est pas membre de l'ASA et donc pas liée par le "gentlemen's agreement», on applique le nouvel art. 35 al. 2 du Code de déontologie de la FMH. Celui-ci prévoit que le médecin est tenu, en sa qualité de membre de la FMH, de prendre à sa charge les honoraires de l'expert mandaté par le Bureau d'expertises de la FMH.

Une couverture d'assurance adéquate permet toutefois de reporter le risque de coûts sur la compagnie d'assurances.

\section{Références}

1 Cf. Hartmann Caroline. Nouveau règlement du Bureau d'expertises extrajudiciaires de la FMH. Bull Med Suisses. 2019;100(38): $1252-3$.

2 Art. 35 du Code de déontologie de la FMH.

3 Voir aperçu des membres de l'ASA, sous www.svv.ch/fr/membres (page consultée le 28 août 2019).
4 Voir www.fmhinsurance.ch, Roth Gygax \& Partner AG, Moosstrasse 2, 3073 Gümligen. 